\title{
Historia breve de las enfermedades tropicales en América
}

\author{
Brief history of tropical diseases in America
}

\author{
Dulce María del Carmen Delgadillo Álvarez' \\ Centro de Investigación y de Estudios Avanzados del Instituto Politécnico Nacional de México \\ dulmadelca@hotmail.com
}

\section{RESUMEN}

La región tropical de la Tierra comprende la zona entre los trópicos de Cáncer y de Capricornio y es en esta franja en donde se localizan países con grandes riquezas naturales pero con economías de ingresos bajos o medios-bajos. Aunado a esto, las características climatológicas de la región son propicias para el desarrollo de organismos patógenos que causan enfermedades graves en la población humana. El término enfermedad tropical, es resultado de un proceso histórico social observado por los europeos durante la conquista y la colonia pues, en el trópico americano, las enfermedades tropicales no existían antes de la llegada de los españoles. Cuando los conquistadores llegaron llevaron consigo patógenos que causaron la muerte de casi el $90 \%$ de la población original siendo las epidemias de viruela, sarampión y gripe las más devastadoras. La conquista del continente fue posible en gran medida por el intercambio biológico entre conquistadores y conquistados y estuvo acompañada de la destrucción de las dos civilizaciones americanas más avanzadas, la azteca en México y la inca en Perú. Durante los siguientes siglos, el intercambio biológico ha sido más amplio; las enfermedades tropicales están ahora presentes en todo el mundo y deben ser atendidas no importando la región del mundo en la que se manifiesten.

Palabras clave: Trópicos, epidemias, patógenos, historia.

\begin{abstract}
The tropical region comprises the area between Cancer and Capricorn tropics where countries with large natural resources and low or lower-middleincome economies are located. Moreover, the region climatic characteristics are conducive to the development of pathogens causing serious diseases on the human population. In the American tropics the term tropical disease is the result of a socialhistorical process observed by Europeans during the conquest and colony periods. Tropical diseases did not exist before the Spaniards arrival. When conquerors arrived they brought pathogens, which caused almost $90 \%$ of the population's death, being the most devastating epidemics: smallpox, measles and flu. The continent conquest was largely made possible by the biological exchange between conquerors and conquered, along with the destruction of two of the most advanced American civilizations: the Aztec in Mexico and the Inca in Peru. During the following centuries, the biological exchange has been extended; tropical diseases are now present throughout the world and must be attended regardless of the world region in which they manifest.
\end{abstract}

Keywords: Tropics, epidemics, pathogens, history.

Historial del artículo:

Recibido: 29 de septiembre de 2015. Aprobado: 25 de noviembre de 2015. Disponible en línea: 30 de diciembre de 2015

1 Licenciada en Biología por la Universidad Nacional Autónoma de México. Maestra en Ciencias en el área de Patología Experimental por el Centro de Investigación y de Estudios Avanzados del Instituto Politécnico Nacional (IPN). Doctora en Ciencias en Biomedicina Molecular en el Centro de Ciencia Aplicada y Tecnología Avanzada del IPN. Auxiliar de Investigación en el dto. de Biomedicina Molecular, Centro de Investigación y de Estudios Avanzados del IPN. Apartado Postal 14-740, 07360 México, DF, México. Teléfono: 57473800 ext. 5013. 


\section{INTRODUCCIÓN}

La región intertropical, tórrida o tropical de la Tierra está comprendida entre los llamados trópico de Cáncer y Trópico de Capricornio. Estas líneas imaginarias son los paralelos ubicados a latitudes de $23^{\circ} 26^{\prime} 14^{\prime \prime}$ en los dos hemisferios, norte y sur, con respecto al Ecuador. Ambas líneas delimitan los puntos más septentrional y meridional en los que el Sol llega a brillar desde el cénit (la vertical del lugar) a mediodía, lo que ocurre cada año entre el 20 y 21 de junio, (para el Trópico de Cáncer), y el 21 y 22 de diciembre (para el Trópico de Capricornio). Los países que atraviesan estos paralelos son, al norte: el territorio no autónomo del Sahara Occidental, Mauritania, Malí, Argelia, Nigeria, Libia, Chad, Egipto, Arabia Saudita, Emiratos Árabes Unidos, Omán, India, Bangladés, Birmania o Myanmar, China, Taiwán, Hawái, México y Bahamas. Y, al sur: Chile, Argentina, Paraguay, Brasil, Namibia, Botswana, Sudáfrica, Mozambique, Madagascar y Australia. La zona tropical ocupa el $20 \%$ de la tierra emergida representando el $40 \%$ de la tierra útil para el hombre pues dada la cantidad de lluvia que cae cada año es buena para la agricultura. También presenta la mayor diversidad ecológica del mundo, tanto en especies vegetales como animales y en ella se ubica más del $40 \%$ de la población mundial aunque, desigualmente distribuida en el territorio (1).

A pesar de la vastedad en riquezas naturales, esta franja geográfica presenta enormes problemas en cuanto a la salud de las personas que la habitan pues posee las características climatológicas adecuadas para el desarrollo de muchos organismos patógenos que causan las llamadas enfermedades tropicales. Con organismos particulares de cada región comprendida en la zona tropical, las enfermedades ocasionadas por éstos son endémicas y afectan a cerca de 1000 millones de personas particularmente de zonas pobres en países poco desarrollados, pues más del $70 \%$ de ellos tienen economías de ingresos bajos o medios-bajos. Además, y aunque las enfermedades tropicales son propias de climas cálidos, son también consecuencia de una salud pública deficiente originada en la pobreza, la ignorancia y la inadecuada organización social (2). Como ejemplo de las consecuencias de esto, se estima que, a nivel mundial, en 2002 hubo 57 millones de muertes, de las que el 10,5\% fueron niños menores de 5 años. La mayoría de estas muertes fueron en países de ingresos bajos o medios-bajos y son atribuidas a enfermedades prevenibles como malaria, diarreas y tétanos entre otras. A todas estas enfermedades se les ha calificado como "enfermedades infecciosas - enfermedades tropicales desatendidas". Otra consecuencia es que, particularmente, en Latino América durante el período 2000 - 2004 se reportaron aproximadamente 58 muertes por 100000 habitantes atribuidas a enfermedades infecciosas lo que significa que estas siguen siendo un gran problema en la región.
Haití por ejemplo presenta la más alta incidencia de tuberculosis así como la mayor resistencia a múltiples drogas contra esa enfermedad. Por otro lado, Brasil reportó en 2006 el 50 \% de casos de dengue de la región (3).

\section{¿Cuál es el origen de estas enfermedades y cómo llegaron a América?}

En general, es difícil conocer la procedencia de cualquier tipo de enfermedad pues para reconocerla en nuestros antepasados debe cumplir con el requisito indispensable de dejar huellas reconocibles en las estructuras anatómicas que se conservan. La paleopatología, es la ciencia que se encarga de estudiar las enfermedades tanto en restos humanos y de animales antiguos, como huesos o momias, como en documentos de culturas ancestrales. De este modo se han encontrado registros de tuberculosis y tifo en materiales preservados de culturas atávicas como la egipcia y la griega (4). En el hombre moderno se ha propuesto que el origen y la dispersión de las enfermedades infecciosas fueron paralelos a la domesticación de animales durante el período Neolítico; sin embargo, la procedencia de este tipo de enfermedades es mucho más antigua que el hombre mismo pues recientemente, con el desarrollo de las técnicas genéticas y moleculares, se ha sugerido que enfermedades modernas como la tuberculosis, la lepra y la treponematosis tienen un origen en el período Paleolítico (figura 1), (5).

El concepto de "enfermedad tropical" fue resultado de un proceso histórico social observado por los europeos durante la época de la conquista y la colonia, ya que, en el trópico americano, las enfermedades tropicales no existían antes de la llegada de los españoles (6). Sin embargo, a lo largo de la historia han hecho falta historiadores con formación científica y científicos con preparación histórica para establecer claramente el papel del nacimiento de la patología tropical. Yendo un poco atrás en la historia del continente americano, se sabe que sus primeros pobladores fueron contingentes de hombres procedentes del noreste de Asia que, aprovechando una pausa interglaciar, durante miles de años cruzaron la cadena de las islas Aleutianas y el estrecho de Bering y fueron ocupando el continente desde Alaska hasta el sur de la Patagonia (7).

Estos primeros grupos de seres humanos encontraron en América un medio favorable para su supervivencia y el pasaje gélido del Estrecho de Bering resultó ser un filtro natural que eliminó gran parte de los organismos patógenos existentes y pocos gérmenes que habían coevolucionado con el hombre lograron filtrarse; de este modo, durante miles de años el espacio americano estuvo aislado de Eurasia y de África y por lo tanto 
libre de la mayor parte de las plagas y enfermedades que devastaron a esos continentes. De hecho, las mortandades previas a 1492 parecen haber sido más por escaseces alimentarias, debido a malas cosechas, que por enfermedades, aunado a esto el hecho de que tampoco había animales domésticos que mantuvieran y transmitieran a los hombres enfermedades de tipo infectocontagioso (7).

Cuando los conquistadores llegaron a América llevaron consigo materiales biológicos que se introdujeron en el Nuevo Mundo para bien y para mal. Ejemplo de esto son las semillas de plantas para reproducir las especies europeas en las nuevas tierras pero que venían acompañadas de semillas que producen malas hierbas además de roedores portadores de gérmenes patógenos. Las nuevas especies encontraron un campo fértil donde propagarse transformándose en malezas y había sido reducida a un $10 \%$ de la cifra original (10).

Los primeros lugares a los que los conquistadores llegaron en América se ubican en las Antillas. Cristóbal Colón realizó el segundo viaje a América entre 1493 y 1496 y trajo consigo el virus de la influenza o gripe porcina; el foco de infección se extendió de la isla de Santo Domingo a las islas vecinas de Puerto Rico, Cuba e incluso las Bahamas. Esta fue la primera epidemia registrada en el nuevo mundo. En la parte continental, además de la influenza, la viruela fue la principal causa de mortalidad de sus habitantes. Se estima que en 1518 un tercio de la población indígena fue exterminada por la viruela. A esto le siguió una epidemia de sarampión entre 1530 y 1531 . En 1546, el genocidio natural continuó con un padecimiento con características de tifus y en 1558 fue nuevamente la gripe. Como consecuencia del encuentro entre

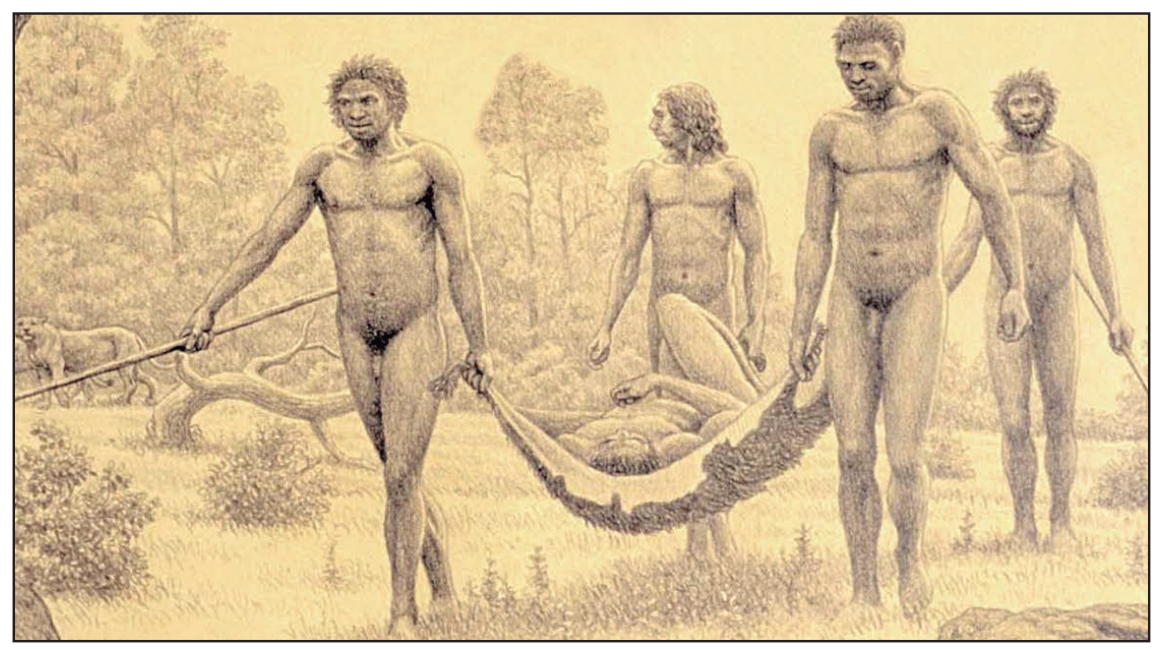

Figura $N^{\circ}$ 1: El Paleolítico; enfermedades como la tuberculosis, lepra y treponematosis tendrían su origen en este período.

Fuente: $\quad$ Museo de la Evolución Humana.

http://www.museoevolucionhumana.com/media/files/Elritodelamuerte.pdf

plagas perturbando el equilibrio ecológico americano $(7,8)$.

En la América precolombina, a pesar de las discrepancias en cuanto al número de pobladores antes de la llegada de los conquistadores, en general se estima que la cantidad de gente que lo habitaba osciló entre 60 y 80 millones de personas. La mayor densidad poblacional estaba en centros urbanos como los de México con cerca de 25 millones y Perú con una cifra de 10 a 15 millones (9). Como se mencionó antes, los pobladores americanos no habían tenido contacto con muchos patógenos y por lo tanto sus defensas inmunológicas eran casi nulas; la conquista los condujo a quedar expuestos a las enfermedades de europeos y africanos tales como la viruela, el sarampión, la gripe o el tifus, la fiebre amarilla o la malaria. Así, para finales del siglo XVI la población americanos y europeos, además de los esclavos africanos, a mediados del siglo XVI había muerto dos tercios de la población indígena inicial de América y su tasa de mortalidad llegó a ser el doble de la que había sufrido Europa durante la peste Negra en el siglo $\operatorname{XIV}(8,10)$. La conquista del continente fue posible en gran medida por el intercambio biológico entre conquistadores y conquistados y estuvo acompañada de la destrucción de las dos civilizaciones americanas más avanzadas, la Azteca en México y la Inca en Perú (11).

Durante los siguientes siglos, el intercambio biológico fue cada vez más amplio debido a factores como los cambios ecológicos entre los que se incluyen el desarrollo de la agricultura y la deforestación, los movimientos poblacionales originados por migraciones de refugiados, guerras y desastres 
naturales, el comercio y los viajes internacionales (12). Todo esto llevo a considerar a la medicina tropical como un factor básico para el desarrollo económico de las grandes potencias mundiales. A principios del siglo XIX, países como Inglaterra, Francia, Bélgica y Holanda entre otros, que poseían colonias en América, África y la India comenzaron a ocuparse de las enfermedades que acababan con sus colonos. Esto, aunado a los avances científicos de finales del siglo XIX y principios del siglo XX dio como resultado la prevención y control de muchas enfermedades infecciosas principalmente en los países desarrollados; sin embargo en países con economías de ingresos medios o medios-bajos han continuado apareciendo brotes de enfermedades infecciosas clasificadas como emergentes y reemergentes (13). Las primeras definidas como infecciones nuevas aparecidas en una población dada en los últimos 30 años, por ejemplo el síndrome de inmunodeficiencia adquirida (VIH/SIDA) y las segundas como aquellas que habiendo existido con anterioridad y que se presumía habían desaparecido o disminuido, comienzan a elevar su incidencia o su alcance geográfico, como la tuberculosis pulmonar o el sarampión (12).

Otro aspecto que tiene un gran impacto en el desarrollo de las enfermedades infecciosas es la evolución de los organismos patógenos como la aparición de cepas de bacterias resistentes a antibióticos y parásitos con una amplia resistencia a los medicamentos con los que se solían controlar. En el caso de los virus muchos de ellos muestran un elevado índice de mutación que les ha permitido producir nuevas variantes. Un claro ejemplo de esto es el virus de la influenza cuyas epidemias anuales son producidas por un desplazamiento antigénico en una cepa que circulaba previamente. Un cambio en un sitio de una proteína de superficie a un sitio antigénico, permite que la nueva variante infecte a personas que ya habían sido infectadas por el mismo virus, pero que, ahora, por el cambio sufrido no es reconocido por el sistema inmune (12).

\section{¿Por qué es tan difícil controlar a las enfermedades tropicales?}

Uno de los aspectos básicos para entender el desarrollo de una enfermedad es conocer su morbilidad, es decir su frecuencia dentro de una población; usualmente se usan las tasas de morbilidad por 100000 habitantes. Para documentar esto es necesario tener sistemas de registro confiables que incluyan la aplicación universal de métodos de diagnóstico de la enfermedad con elevado nivel de acierto y la cobertura de grupos estadísticamente representativos, que permitan la extrapolación de datos a la población general. Este tipo de información comenzó a tenerse, como mencionamos previamente, a principios del siglo XIX principalmente en Inglaterra y los países escandinavos. Ya a mediados del mismo siglo se habían sumado a esta práctica Francia, Alemania, Italia y Suiza. Siglo y medio después, muchos países ubicados en la franja tropical del planeta aún no cuentan con este tipo de información por lo que la documentación cuantitativa necesaria para conocer con una aproximación razonable las variaciones en frecuencia de un padecimiento existen desde hace muy poco y sólo para algunos países del mundo (4).

Otra razón para que el control de las enfermedades infecciosas no sea tan exitoso como se desea es que, a pesar del desarrollo de nuevas tecnologías encaminadas al diseño y elaboración de medicamentos y vacunas contra los patógenos, los avances no han sido sustanciosos. Cada vez es más frecuente encontrar resistencia contra los antibióticos y la creación de nuevos es escasa. En relación a las vacunas, aún no se ha logrado obtener alguna que sea realmente efectiva contra el VIH/SIDA o la malaria (14). Por otro lado, luego de que un fármaco o una vacuna han sido creados y aprobados por las autoridades correspondientes para su uso o aplicación en comunidades humanas, su implementación en las poblaciones que lo necesitan puede tardar en promedio aproximadamente 10 años. Un ejemplo de esto es la producción, distribución y aplicación de la vacuna contra la Hepatitis $B$, que en países desarrollados llevó 2 a 5 años mientras que en países de ingresos medios fue de 8 años y en países de bajo ingreso fue de casi 20 años (15).

Otra causa que obstaculiza el control de las enfermedades tropicales está dada por los fenómenos climáticos. Se han identificado regiones geográficas, comprendidas en la franja tropical donde las condiciones ambientales pueden incrementar la incidencia de transmisión de las enfermedades infecciosas como, entre otras, el cólera y el dengue. Para el primero, las temperaturas más cálidas pueden promover la proliferación bacteriana y las inundaciones la contaminación de las fuentes de agua. En el caso del dengue, en condiciones de sequía, los reservorios de agua domésticos son los sitios de reproducción de los mosquitos vectores y cuando la precipitación es elevada, éstos encuentran un ambiente más favorable para su crianza (16).

\section{¿Cuáles han sido los logros en cuanto al control de las enfermedades infecciosas?}

La Organización Mundial de la Salud (OMS) surgida en 1948, después de la segunda guerra mundial, creó organismos para el control de muchas endemias mundiales orientándose hacia el control de algunas enfermedades inmunoprevenibles. De todas ellas la única enfermedad controlada a nivel mundial por vacunas ha sido la viruela, erradicada en 1977 y la poliomielitis infantil reportó un último caso en América 
en 1991 por lo que, en 1994, la Comisión Internacional para la Certificación de la Erradicación de la Poliomielitis declaró que la circulación del virus salvaje de la polio se había interrumpido en el continente (13, 17). Sin embargo, la enfermedad persiste en Camerún, Guinea Ecuatorial, Pakistán, Siria, Afganistán, Etiopía, Iraq, Israel, Somalia y Nigeria. Considerando esto, la Organización Panamericana de Salud declaró en 2014 que el poliovirus salvaje sigue constituyendo una Emergencia de Salud Pública de Importancia Internacional o ESPII y recomienda mantener los niveles requeridos de cobertura de vacunación contra polio así como continuar con la vigilancia adecuada de la parálisis flácida aguda, para mantener el estatus de la región como libre de esa enfermedad. Sugiere además a todo viajero que salga del continente americano y se dirija a los países que actualmente presentan la infección que se inmunice previamente a su viaje $(14,18)$.

Por otro lado, en el caso de la fiebre amarilla que contaba con dos tipos, el urbano y el selvático, se ha logrado erradicar al primero y actualmente sólo se tiene a la forma selvática en algunos países de Suramérica como Brasil, Bolivia y Perú (13).

En el mundo se han reportado más de 1400 organismos patógenos que causan enfermedades en el humano; más de 300 son de importancia clínica por lo que es relevante compilar toda la información acerca de su diagnosis, epidemiología y terapias para crear herramientas que permitan al personal médico y a las autoridades sanitarias tomar decisiones informadas para su control $y$, de ser posible, su erradicación. Con los datos compilados se han realizado estudios encaminados a la obtención de un mapa mundial de las enfermedades, esto es conocer su distribución geográfica. Estos mapas permiten visualizar rápidamente la extensión y magnitud de los problemas de salud pública $y$, a pesar de que la idea no es del todo nueva hoy se cuenta con bases de datos que proporcionan información amplia y confiable acerca de las enfermedades que afectan al hombre. Un ejemplo de esto es GIDEON (Global Infectious Disease and Epidemiology Online Network), una base de datos que compila la información de más de 350 enfermedades infecciosas de 231 países. Empleando esta herramienta además de otras bases de datos y de información de la literatura Hay et al. (2013) reportaron un mapa en el que la distribución geográfica de 347 enfermedades de importancia clínica recayó mayoritariamente en la franja comprendida entre los Trópicos de Cáncer y de Capricornio, es decir en la zona tropical del planeta (19).

Posteriormente, el mismo grupo de trabajo realizó otro estudio en el que se priorizaron las enfermedades de acuerdo a su morbilidad y mortalidad para determinar objetivamente su impacto. Sin sorpresa se encontró que las enfermedades con más alta prioridad son la malaria, VIH y tuberculosis seguidas de enfermedades transmitidas por contaminación de comida y agua, como el cólera y la esquistosomiasis, además de la tripanosomiasis, la filariasis, las enfermedades transmitidas por contaminación del suelo, como la ascariasis o por mosquitos vectores como chikungunya, dengue y la fiebre amarilla entre otras (20). Todas estas son enfermedades tropicales desatendidas.

\section{CONCLUSIONES}

Las enfermedades tropicales desatendidas son un problema grave dentro de la región que las comprende y que es, lamentablemente, la zona con la población humana de ingresos bajos o mediosbajos. En contraste con una riqueza ecológica evidente, la zona tropical posee las características más desafortunadas para el control y la erradicación de patógenos que demeritan y acaban con la vida de sus pobladores. Contrariamente a lo que se puede pensar, las enfermedades tropicales desatendidas no son un problema local, es decir de la zona tropical, pues la cada vez mayor globalización y los cambios climáticos afectan al mundo en general y necesitan ser atendidas con políticas multidimensionales y multisectoriales. La historia nos ha enseñado que existen enfermedades reemergentes que se habían considerado erradicadas, además de aquellas que son emergentes y de las que sabe poco. Absolutamente todas las enfermedades deben ser atendidas no importando la región del mundo en las que se manifiesten. Esto por la simple razón de que no sabemos qué caminos puedan llevarnos a ellas o las traigan a nosotros sin apenas notarlo.

\section{REFERENCIAS BIBLIOGRÁFICAS}

1. Wikipedia [homepage in Internet]. Trópico de Cáncer y Trópico de Capricornio. [actualizada 30 noviembre 2015; consultada 3 diciembre 2015]. Disponible en: https//es.wikipedia.org

2. World Health Oorganization. [homepage in intenet]. Tropical diseases. [actualizada 2015; consultada julio 2015]. Disponible en: www.who. int/tropics/tropical_diseases/es/

3. Mitra A, Rodríguez-Fernandez G. Latin America and the Caribbean: Assessment of the Advances in Public Health for the Achievement of the Millennium Development Goals. Int J Environ Res Public Health. 2010; 7: 2238-2255.

4. Pérez-Tamayo R. Enfermedades viejas $y$ enfermedades nuevas. México D.F.: Siglo XXI; 1998.

5. Trueba G, Dunthorn M. Many Neglected Tropical 
Diseases May Have Originated in the Paleolithic or Before: New Insights from Genetics. PLoS Negl Trop Dis. 2012; 6(3): 1-4.

6. Carrada-Figueroa G. Importancia de las enfermedades tropicales en México y en el mundo. Salud en Tabasco. 1995; 1(4): 119-122.

7. Cordero del Campillo M. Las grandes epidemias en la América colonial. Archivos de Zootecnia. 2001; 50 (192): 597-612.

8. Muscar Benasayag EF. El Nuevo Mundo, dos encuentros, principales impactos: Períodos precolombino y colonial. Anales de Geografía de la Universidad Complutense. 1992; 12: 157-164.

9. Malamud, C. Historia de América, 2a. ed., Madrid: Alianza Editorial; 2014.

10. Guerra F. Origen de las epidemias en la conquista de América. Quinto centenario. 1988; 14: 43-51.

11. Maddison A. La economía de occidente y la del resto del mundo en el último milenio. Revista de Historia Economica. 2004; 2: 308.

12. Riverón C. Enfermedades emergentes y reemergentes: un reto al siglo XXI. Rev Cubana Pediatr. 2002; 74(1): 7-22.

13. Maguiña V. Historia de la infectología y la medicina tropical y su importancia en Latinoamérica. Diagnóstico. 2000; 38 (5).
14. Dye C. After 2015: infectious diseases in a new era of health and development. Philos Trans R Soc Lond., B, Biol Sci. 2014; B369:20130426: 1-9.

15. Brooks A, Smith TA, de Savigny $D$, Lengeler C. Implementing new health interventions in developing countries: why do we lose a decade or more? BMC Public Health. 2012; 12(683): 1-17.

16. Chretien J-P, Anyamba A, Small J, Britch S, Sanchez JL, Halbach AC, et al. Global climate Anomalies and Potential Infectious Disease Risks:2014-2015. PLoS Currents. 2015; 26(7). Disponible en http:// www.ncbi.nlm.nih.gov/pubmed/25685635

17. Informe de parálisis flácida aguda año 2011. Unidad de Vigilancia, Depto de Epidemiología, Ministerio de Salud, Gobierno de Chile. 2011.

18. XXI Reunión del Grupo Técnico Asesor sobre Enfermedades Prevenibles por Vacunación de la Organización Panamericana de la Salud. Informe final. Quito, Ecuador, 2013.

19. Hay SI, Battle KE, Pigott DM, Smith DL, Moyes $\mathrm{CL}$, Bhatt $\mathrm{S}$, et al. Global mapping of infectious disease. Philos Trans R Soc Lond., B, Biol Sci. 2013; B368:20120250: 1-11.

20. Pigott DM, Howes RE, Wiebe A, Battle KE, Golding N, Gething PW, et al. Prioritising Infectious Disease Mapping. PLoS Negl Trop Dis. 2015; 9(6): 1-21. 